\title{
A ROSAT PSPC X-ray survey of the Small Magellanic Cloud ${ }^{\star}$
}

\author{
P. Kahabka ${ }^{1,2}$, W. Pietsch ${ }^{3}$, M.D. Filipović $\dot{c}^{3,4,5}$, and F. Haberl ${ }^{3}$ \\ ${ }^{1}$ Astronomical Institute, University of Amsterdam, Kruislaan 403, NL-1098 SJ Amsterdam, The Netherlands \\ ${ }^{2}$ Center for High Energy Astrophysics, University of Amsterdam, Kruislaan 403, NL-1098 SJ Amsterdam, The Netherlands \\ ${ }^{3}$ Max-Planck-Institut für extraterrestrische Physik, D-85740 Garching bei München, Germany \\ ${ }^{4}$ University of Western Sydney, Nepean, P.O. Box 10, Kingswood NSW 2747, Australia \\ ${ }^{5}$ Australia Telescope National Facility, CSIRO, P.O. Box 76, Epping NSW 2121, Australia
}

Received May 25; accepted December 7, 1998

\begin{abstract}
We present the results of a systematic search for point-like and moderately extended soft $(0.1-2.4 \mathrm{keV})$ $\mathrm{X}$-ray sources in a raster of nine pointings covering a field of $8.95 \mathrm{deg}^{2}$ and performed with the ROSAT PSPC between October 1991 and October 1993 in the direction of the Small Magellanic Cloud (SMC). We detect 248 objects which we include in the first version of our SMC catalogue of soft X-ray sources. We set up seven source classes defined by selections in the count rate, hardness ratio and source extent. We find five high luminosity super-soft sources (1E 0035.4 - 7230, 1E 0056.8 - 7146, RX J0048.4 - 7332, RX J0058.6 - 7146 and RX J0103 - 7254), one low-luminosity super-soft source RX J0059.6 - 7138 correlating with the planetary nebula L357, 51 candidate hard X-ray binaries including eight bright hard X-ray binary candidates, 19 supernova remnants (SNRs), 19 candidate foreground stars and 53 candidate background active galactic nuclei (and quasars). We give a likely classification for $\sim 60 \%$ of the catalogued sources. The total count rate of the detected point-like and moderately extended sources in our catalogue is $6.9 \pm$ $0.3 \mathrm{~s}^{-1}$, comparable to the background subtracted total rate from the integrated field of $\sim 6.1 \pm 0.1 \mathrm{~s}^{-1}$.
\end{abstract}

Key words: catalogues - Magellanic Clouds - X-rays: stars - X-rays: galaxies — ISM: supernova remnants (SNRs)

\footnotetext{
Send offprint requests to: ptk@astro.uva.nl

* Table 1 is also available in electronic from at the CDS via anonymous ftp to cdsarc.u-strasbg.fr (130.79.128.5) or via http://cdsweb.u-strasbg.fr/Abstract.html
}

\section{Introduction}

The SMC was the subject of several recent high resolution surveys in different wavelength bands. A CO survey of the SMC was produced by Rubio et al. (1993a,b). Recent $\mathrm{H} \alpha$ surveys were performed by Coarer et al. (1993) and Caplan et al. (1996) (see also Davies et al. 1976). At radio frequencies a neutral Hydrogen survey at $1.4 \mathrm{GHz}$ (Staveley-Smith et al. 1997) and continuum surveys at 1.4 and $2.3 \mathrm{GHz}$ (Filipović et al. in preparation) were made with the Compact Array of the Australia Telescope National Facility. Also, Parkes radio surveys of the SMC were undertaken by Filipović et al. (1997).

Early X-ray surveys were performed with the Einstein satellite (Seward \& Mitchell 1981; Inoue et al. 1983; Bruhweiler et al. 1987; Wang \& Wu 1992). Wang \& Wu (1992) found 20 sources from a sample of 70 sources to be intrinsic to the SMC. Filipović et al. (1998) compared the Parkes radio catalogue with the ROSAT PSPC catalogue presented in this paper and found 27 sources common to these two surveys.

Our aim in this paper is to present the first-pass catalogue of ROSAT X-ray sources in the SMC region with their basic properties and classification. This catalogue is given in Table 1 . We classify about $60 \%$ of the sources by making use of X-ray spectral parameters and correlations with optical, radio and other X-ray catalogues. Each source class is discussed separately in terms of statistical properties.

\section{Observations and data analysis}

The observations used in this analysis were carried out with the PSPC detector on-board the ROSAT observatory during nine pointed observations between 8 October 1991 and 14 October 1993. The satellite, its X-ray telescope (XRT) and the focal plane detector (PSPC) used were discussed in detail by Trümper (1983) and Pfeffermann et al. 
(1986). The pointings were performed in a raster covering the Optical Bar and the Eastern Wing of the SMC quite homogeneously (cf. Fig. 1, Fig. 2 and Table 1 in Kahabka \& Pietsch 1996, herafter Paper I). A search for unresolved and moderately extended sources has been conducted on the data obtained from these fields (Table 2).

Table 2. Fields used for source search in the SMC Survey

\begin{tabular}{llccr}
\hline $\begin{array}{l}\text { Point. } \\
\text { (Pap. I })\end{array}$ & $\begin{array}{l}\text { Sequence } \\
\text { Number }\end{array}$ & $\begin{array}{c}\text { RA } \\
(\mathrm{J} 2000.0)\end{array}$ & $\begin{array}{c}\text { Dec } \\
(\mathrm{J} 2000.0)\end{array}$ & $\begin{array}{r}\text { Expos. } \\
(\mathrm{ksec})\end{array}$ \\
\hline A1 & $600195 \mathrm{p}-0$ & $0^{\mathrm{h}} 58^{\mathrm{m}} 12.0^{\mathrm{s}}$ & $-72^{\mathrm{d}} 16^{\mathrm{m}} 48^{\mathrm{s}}$ & 16.6 \\
A2 & $600195 \mathrm{p}-1$ & $0^{\mathrm{h}} 58^{\mathrm{m}} 12.0^{\mathrm{s}}$ & $-72^{\mathrm{d}} 16^{\mathrm{m}} 48^{\mathrm{s}}$ & 9.4 \\
B1 & $600196 \mathrm{p}-0$ & $0^{\mathrm{h}} 50^{\mathrm{m}} 12.0^{\mathrm{s}}$ & $-73^{\mathrm{d}} 13^{\mathrm{m}} 48^{\mathrm{s}}$ & 1.3 \\
B2 & $600196 \mathrm{p}-1$ & $0^{\mathrm{h}} 50^{\mathrm{m}} 12.0^{\mathrm{s}}$ & $-73^{\mathrm{d}} 13^{\mathrm{m}} 48^{\mathrm{s}}$ & 22.2 \\
C & $600197 \mathrm{p}$ & $1^{\mathrm{h}} 13^{\mathrm{m}} 24.0^{\mathrm{s}}$ & $-72^{\mathrm{d}} 49^{\mathrm{m}} 12^{\mathrm{s}}$ & 21.5 \\
D & $600452 \mathrm{p}$ & $1^{\mathrm{h}} 05^{\mathrm{m}} 55.2^{\mathrm{s}}$ & $-72^{\mathrm{d}} 33^{\mathrm{m}} 36^{\mathrm{s}}$ & 14.2 \\
E & $600453 \mathrm{p}$ & $0^{\mathrm{h}} 54^{\mathrm{m}} 28.7^{\mathrm{s}}-72^{\mathrm{d}} 45^{\mathrm{m}} 36^{\mathrm{s}}$ & 17.6 \\
F1 & $600454 \mathrm{p}-0$ & $0^{\mathrm{h}} 42^{\mathrm{m}} 55.2^{\mathrm{s}}-73^{\mathrm{d}} 38^{\mathrm{m}} 24^{\mathrm{s}}$ & 9.7 \\
F2 & $600454 \mathrm{p}-1$ & $0^{\mathrm{h}} 42^{\mathrm{m}} 55.2^{\mathrm{s}}-73^{\mathrm{d}} 38^{\mathrm{m}} 24^{\mathrm{s}}$ & 8.3 \\
G1 & $600455 \mathrm{p}-0$ & $1^{\mathrm{h}} 01^{\mathrm{m}} 16.7^{\mathrm{s}}-71^{\mathrm{d}} 49^{\mathrm{m}} 12^{\mathrm{s}}$ & 3.6 \\
G2 & $600455 \mathrm{p}-1$ & $1^{\mathrm{h}} 01^{\mathrm{m}} 16.7^{\mathrm{s}}-71^{\mathrm{d}} 49^{\mathrm{m}} 12^{\mathrm{s}}$ & 1.7 \\
G3 & $600455 \mathrm{p}-2$ & $1^{\mathrm{h}} 01^{\mathrm{m}} 16.7^{\mathrm{s}}-71^{\mathrm{d}} 49^{\mathrm{m}} 12^{\mathrm{s}}$ & 4.6 \\
G4 & $600455 \mathrm{p}-3$ & $1^{\mathrm{h}} 01^{\mathrm{m}} 16.7^{\mathrm{s}}-71^{\mathrm{d}} 49^{\mathrm{m}} 12^{\mathrm{s}}$ & 4.1 \\
X0 & $400299 \mathrm{p}-0$ & $0^{\mathrm{h}} 37^{\mathrm{m}} 19.2^{\mathrm{s}}-72^{\mathrm{d}} 14^{\mathrm{m}} 24^{\mathrm{s}}$ & 5.1 \\
X1 & $400299 \mathrm{p}-1$ & $0^{\mathrm{h}} 37^{\mathrm{m}} 19.2^{\mathrm{s}}-72^{\mathrm{d}} 14^{\mathrm{m}} 24^{\mathrm{s}}$ & 1.7 \\
X2 & $400299 \mathrm{p}-2$ & $0^{\mathrm{h}} 37^{\mathrm{m}} 19.2^{\mathrm{s}}-72^{\mathrm{d}} 14^{\mathrm{m}} 24^{\mathrm{s}}$ & 2.3 \\
Y1 & $400300 \mathrm{p}-0$ & $0^{\mathrm{h}} 58^{\mathrm{m}} 33.5^{\mathrm{s}}-71^{\mathrm{d}} 36^{\mathrm{m}} 00^{\mathrm{s}}$ & 5.2 \\
Y2 & $400300 \mathrm{p}-1$ & $0^{\mathrm{h}} 58^{\mathrm{m}} 33.5^{\mathrm{s}}-71^{\mathrm{d}} 36^{\mathrm{m}} 00^{\mathrm{s}}$ & 7.2 \\
Y3 & $400300 \mathrm{p}-2$ & $0^{\mathrm{h}} 58^{\mathrm{m}} 33.5^{\mathrm{s}}-71^{\mathrm{d}} 36^{\mathrm{m}} 00^{\mathrm{s}}$ & 7.1 \\
\hline
\end{tabular}

A sophisticated detection procedure was applied to the SMC survey. Each pointed observation has been analyzed with three detection methods (local, map and maximum likelihood, cf. Zimmermann et al. 1994). These detection procedures were applied to the data of single pointings given in Table 2. Data with the same pointing direction have been merged to one data set. The analysis was performed in the five energy channel ranges Soft $=($ channel $11-41$, $0.1-0.4 \mathrm{keV})$, Hard $=($ channel $52-201,0.5-2.1 \mathrm{keV})$, Hard $1=($ channel $52-90,0.5-0.9 \mathrm{keV})$, Hard $2=($ channel $91-201,0.9-2.0 \mathrm{keV})$ and Broad $(0.1-2.4 \mathrm{keV})$. The five source lists were merged to one final source list taking only detections at an off-axis angle $\leq 45^{\prime}$ into account. This list comprises the source catalogue published in this paper. The maximum likelihood algorithm was used to determine the final source position, the counts in five energy bands and the source extent. A one-dimensional energy and position dependent Gaussian distribution (cf. Zimmermann et al. 1994) was applied in order to obtain the source extent. The source extent $(E x t)$ is given as the Gaussian $\sigma_{\text {Gauss }}$

$E x t=\sigma_{\mathrm{G} \text { auss }}=F W H M_{\mathrm{G} \text { auss }} / 2.35$.
Hardness ratios $H R 1$ and $H R 2$ were calculated from the counts in the bands as $H R 1=(H-S) /(H+S)$ and $H R 2=(H 2-H 1) /(H 1+H 2)$. The existence likelihood ratio and the extent likelihood ratio was calculated according to Cash (1979) and Cruddace et al. (1988).

\section{The catalogue}

We selected for our final source catalogue only detections with an existence likelihood ratio $L H_{\text {exist }}>10$, which is equal to a probability of existence $P \sim(1-$ $\left.\exp \left(-L H_{\text {exist }}\right)\right) \sim\left(1-4.510^{-5}\right)$. We give the value for the extent only in case the extent likelihood ratio is $L H_{\text {extent }} \geq 10$.

A $90 \%$ source error radius was calculated, adding quadratically a $5^{\prime \prime}$ systematic error.

$P_{\mathrm{e}}=2.1 \times \sqrt{x_{\text {err }}^{2}+y_{\text {err }}^{2}+\left(5^{\prime \prime}\right)^{2}}$.

The positional error derived for large off-axis angles $\Delta \gtrsim$ $30^{\prime}$ may be somewhat underestimated due to the asymmetry of the point-spread-function. But the positional error should not be larger than $\sim 1^{\prime}$.

We catalogued all point-like and moderately extended sources found in this survey in Table 1. A small fraction $(\sim 9 \%)$ of catalogued sources may be false (artifacts due to the PSPC detector structure). The source positions were not corrected with respect to the positions of known standard sources (e.g. foreground stars or background active galactic nuclei (AGNs)) because it may easily introduce improper shifts in position. Column 1 gives the source catalogue number and Col. 2 the ROSAT source name. In Cols. 3 and 4 we list source positions, the right ascension (RA) and declination (Dec) for the epoch J2000 with the $90 \%$ confidence positional uncertainty (Col. 5). Column 6 gives the total count rate (with the $1 \sigma$ errors). We list the soft $(H R 1)$ and the hard (HR2) hardness ratio (with $1 \sigma$ errors) in Cols. 7 and 8 , while in Col. 9 we present the source extent in cases where the likelihood ratio for extent is greater than 10. In Col. 10 is listed the likelihood ratio of existence ( $\left.L H_{\text {exist }}\right)$ and in Col. 11 the off-axis angle $(\Delta)$ for the detection with the highest $L H_{\text {exist }}$ from different bands. Columns 12 and 13 give the Einstein index (from the Wang \& Wu 1992, catalogue) and the distance to the Einstein source. Columns 14, 15 and 16 list the stellar type, the magnitude of the Simbad identified star and the distance to the Simbad star. In Col. 17 we list the source class according to our classification scheme (Sect. 5, Table 3). In addition four sources are classified as SNRs although they have a smaller extent likelihood ratio as required from the classification. However, they coincide with SNRs detected with Einstein. In Col. 18 we give a refined classification for candidate AGN and hard X-ray binary. In Col. 19 we give some notes on either radio or optical identifications. 
For the hard X-ray binary and the AGN class we have refined the classification scheme. We have taken the local Hydrogen column into account and from simulations of power-law slope -2.0 and -2.6 AGN spectra we have predicted hardness ratios which we compared with the measured hardness ratios. In case the classification was not unique we introduced the class AB in Table 1, Col. 18.

In Fig. 1 we show the spatial distribution of all 248 detections in the direction of the SMC overlaid on a merged exposure image. Only the central $45^{\prime}$ of the field of view are considered for source detection.

\section{The survey}

The X-ray survey of the SMC covers a field of $8.95 \mathrm{deg}^{2}$ considering the used $45^{\prime}$ field of view of each pointed observation. From the merged $(0.1-2.4) \mathrm{keV}$ image corrected for exposure we derive a total count rate of $53.2 \mathrm{~s}^{-1}$. The mean exposure of this image is $7500 \mathrm{~s}$ and the maximum $22000 \mathrm{~s}$. We created a merged background image of the same field and subtracted the background rate image from the count rate image. We find an excess rate of $6.1 \pm 0.1 \mathrm{~s}^{-1}$. In comparison the total source count rate we derive from our catalog of 248 point-like and moderately extended sources is $6.9 \pm 0.3 \mathrm{~s}^{-1}$. Both rates are within the $2 \sigma$ errors identical and are in agreement with the finding from the ROSAT All-Sky Survey (RASS) data that the residual count rate of an extended emission component (a hot gas) is small. Kahabka \& Pietsch (1993) find an excess count rate of $\sim 0.7 \mathrm{~s}^{-1}$ for the $8 \times 8 \mathrm{deg}^{2}$ RASS field after subtracting a smooth background component and the source contribution. This result may be compared with the finding of Irwin \& Sarazin (1998). They found that the integrated X-ray emission and colors of X-ray faint galaxies (group 1 galaxies) to which also M 31 and the SMC are belonging can be explained as the integrated emission from (low-mass) X-ray binaries.

\section{Source classes}

The X-ray spectral characteristics of the catalogued sources are given as two hardness ratios (the soft hardness ratio - $H R 1$ and the hard hardness ratio - HR2), the source extent (assuming a Gaussian model) and the count rates in a spectrally soft and hard band. This information is used to characterize a source and to allow a source classification. We previously applied some selections to this catalogue in order to derive the sample of super-soft X-ray sources of the SMC (Kahabka et al. 1994). In Kahabka \& Pietsch (1996) we applied another set of selections in order to derive the sample of spectrally hard X-ray binary candidates with luminosities in excess of $310^{35} \mathrm{erg} \mathrm{s}^{-1}$. These subsets comprise only a small number of sources (four supersoft and
$7-13$ hard). The majority of sources (209) have not been classified previously.

In a recent paper Filipović et al. (1998) presented results of a comparison between the ROSAT PSPC catalogue presented here and the Parkes catalogue of sources towards the SMC at radio frequencies $(1.42,2.45,4.75$, 4.85 and $8.55 \mathrm{GHz}$ ). They found 27 sources in common to both surveys (cf. Table 3 in their paper). These include 14 SNRs (with two SNR candidates), eight background sources and three $\mathrm{H}$ II regions.

We now define other classes, set up the selection criteria, identify the members, derive the relevant distributions of the source properties and discuss the implications in terms of population studies. The positional distribution of the classified sources using criteria established here (Table 3) is shown in Fig. 2.

\subsection{The high luminosity super-soft sources}

This class was extensively discussed in Kahabka et al. (1994) and in Paper I. Applying the strict selection criteria outlined in these papers $(H R 1+\delta H R 1<-0.8$ and count rate $>0.015 \mathrm{~s}^{-1}$ ) we obtain candidates classified as class $=$ Sl in Table 1. Sources Nos. 20, 62, 133, 135 and 181 were discussed in Paper I. Sources Nos. 6, 112 and 173 coincide with struts of the PSPC detector system and are classified as possible detector artifacts.

\subsection{The low luminosity super-soft sources}

Low luminosity super-soft sources are not yet established as a new class of objects, but they are expected to exist e.g. as hot central stars of planetary nebulae ( $\mathrm{PNe})$ which, if they are on the cooling track, can attain luminosities in the range $10^{34}-10^{36} \mathrm{erg} \mathrm{s}^{-1}$. This would translate into $P S P C$ count rates for sources in the SMC of $\sim 710^{-5}-$ $710^{-3} \mathrm{~s}^{-1}$ i.e. below our threshold of $0.015 \mathrm{~s}^{-1}$ for high luminosity super-soft sources.

There are three objects which are fulfilling these criteria and they are classified as class $=\mathrm{Sw}$ in Table 1 . The source No. 38 is found at a large off-axis angle of $43^{\prime}$ and is extended (Gaussian $\sigma=214^{\prime \prime}$ ). This may indicate either diffuse structure or an artifact. The source No. 141 coincides with a Simbad star of 17.5 mag and with the planetary nebula L357 in the catalogue of Meyssonnier \& Azzopardi (1993). It is therefore a real candidate. Source No. 243 is found at an off-axis angle of $20^{\prime}$ and coincides with the inner PSPC support structure and may be an artifact. This leaves us with one firm low-luminosity supersoft source No. 141 (RX J0059.6 - 7138).

\subsection{The "stronger" hard X-ray binary candidates}

The sample of X-ray binary candidates with luminosities in excess of $310^{35} \mathrm{erg} \mathrm{s}^{-1}$ has been given in Paper I. 


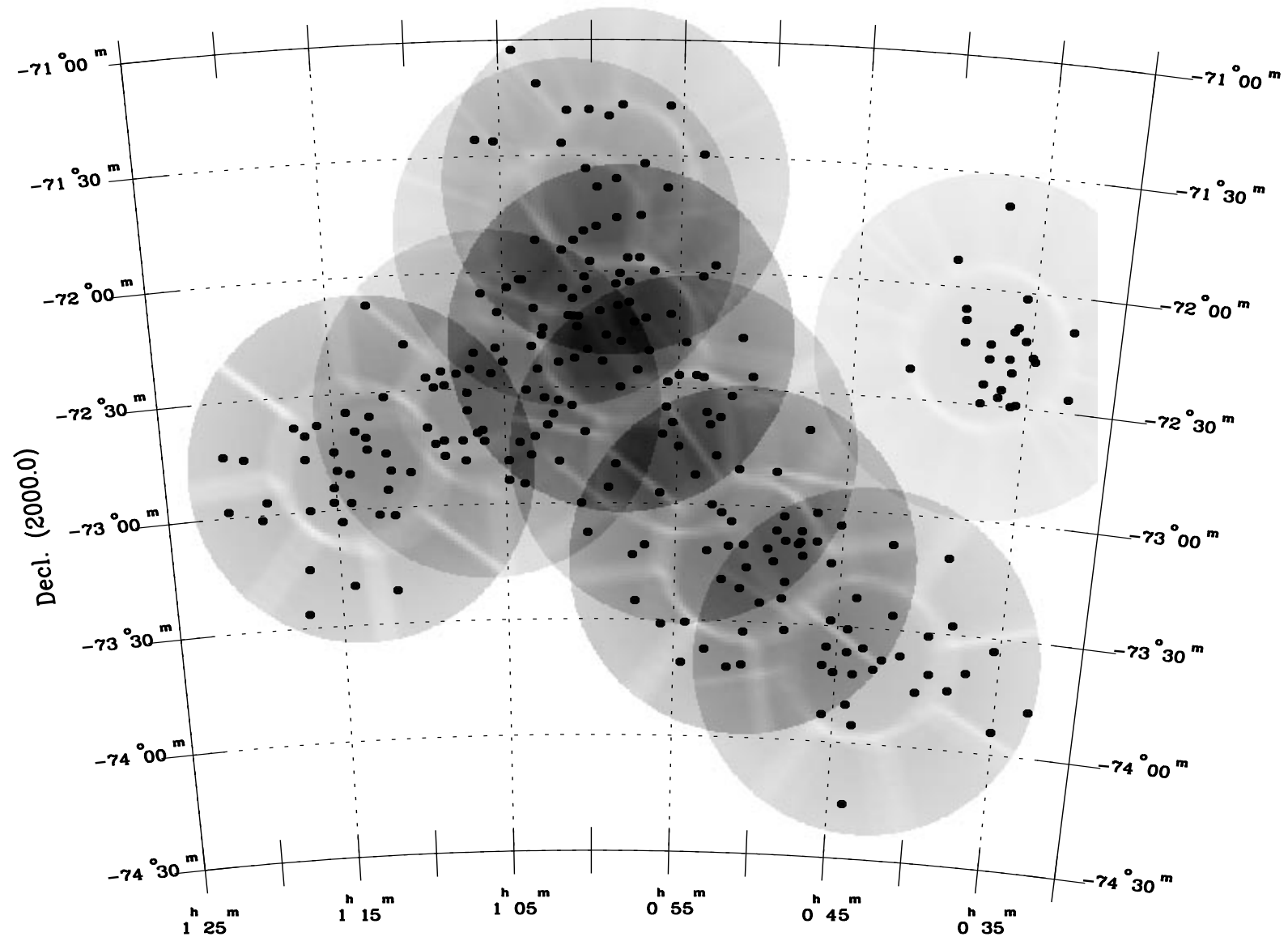

R.A. (2000.0)

Fig. 1. Distribution of all X-ray detections in the field of the SMC drawn over integrated exposure map

Table 3. Definition of the source classes and the selection criteria

\begin{tabular}{|c|c|c|c|c|c|}
\hline \multicolumn{2}{|r|}{ Source Class } & \multicolumn{4}{|c|}{ Selection Criteria } \\
\hline & & $\begin{array}{l}\text { Count Rate } \\
\qquad\left(\mathrm{s}^{-1}\right)\end{array}$ & $H R 1$ & $H R 2$ & $\begin{array}{c}\text { Extent } \\
\text { Likelihood }\end{array}$ \\
\hline $\mathrm{Sl}$ & High luminosity super-soft sources & $>0.015$ & $H R 1+\delta H R 1<-0.8$ & & \\
\hline $\mathrm{Sw}$ & Low luminosity super-soft sources & $<0.015$ & $H R 1+\delta H R 1<-0.8$ & & \\
\hline $\mathrm{Bl}$ & Stronger hard X-ray binaries & $>0.015$ & $H R 1-\delta H R 1>+0.5$ & $H R 2-\delta H R 2>+0.3$ & $<50$ \\
\hline $\mathrm{Bw}$ & Weaker hard X-ray binaries & $<0.015$ & $H R 1-\delta H R 1>+0.5$ & $H R 2-\delta H R 2>+0.3$ & $<50$ \\
\hline $\mathrm{R}$ & SNRs and extended structure & & $H R 1-\delta H R 1>-0.8$ & & $>50$ \\
\hline $\mathrm{F}$ & Foreground stars & & $H R 1+\delta H R 1<+0.5$ & $H R 2-\delta H R 2>-0.8$ & $<50$ \\
\hline A & Background objects (AGNs) & & $H R 1-\delta H R 1>+0.5$ & $H R 2+\delta H R 2<+0.3$ & $<50$ \\
\hline $\mathrm{D}$ & Possible artifacts & & & & \\
\hline
\end{tabular}

Remark: additional classes from refined classification: $\mathrm{AB}=\mathrm{AGN}$ or hard X-ray binary source and $\mathrm{H}=\mathrm{H} \mathrm{I}$ region. 


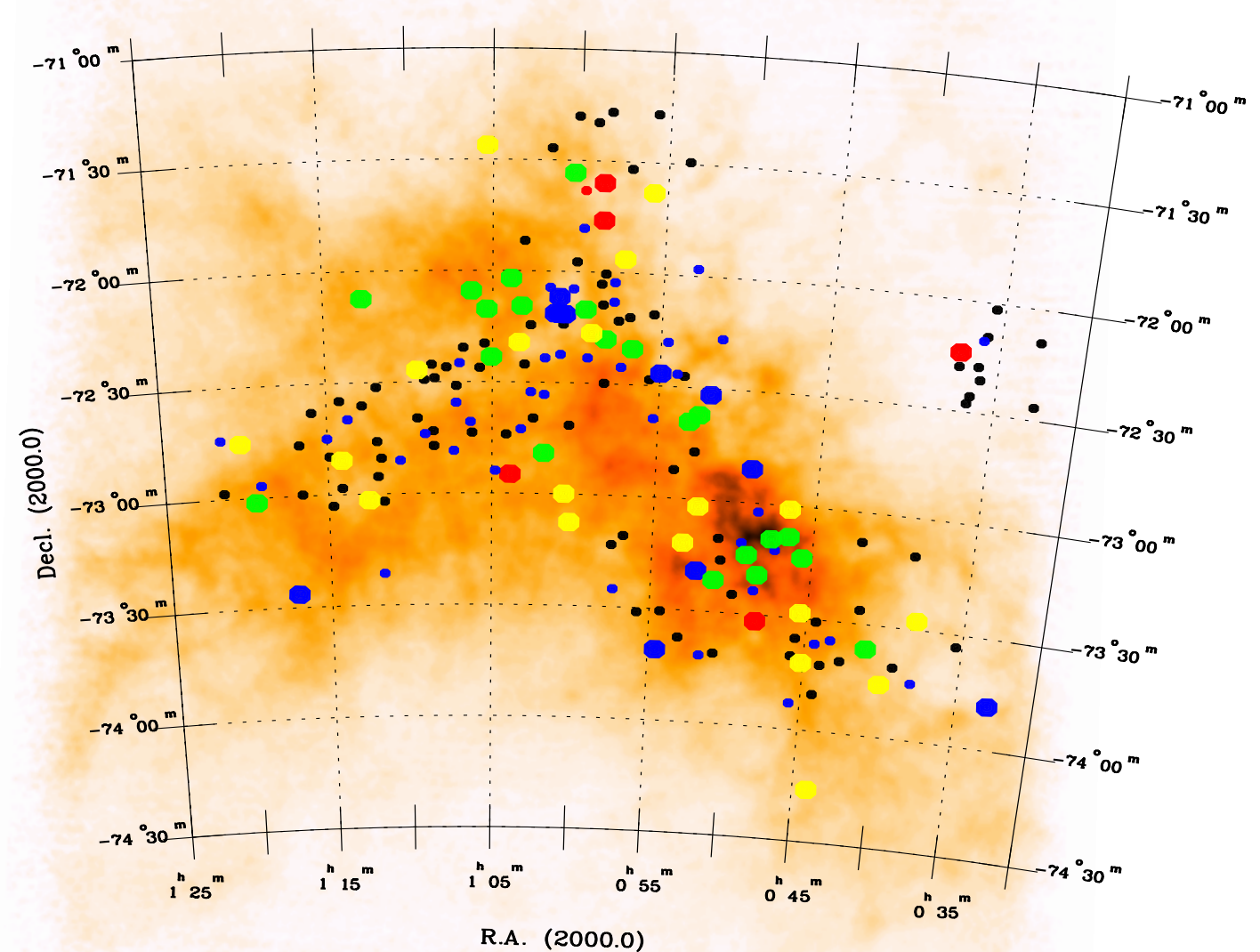

Fig. 2. Distribution of classified X-ray detections (this work) in the field of the SMC plotted over high resolution H I image of the SMC (from Stanimirovic et al. 1999). Selected classes are: super-soft sources (red), hard X-ray binaries (blue), AGNs (black), SNRs (green) and foreground stars (yellow). Big/small circles are drawn for the high/low luminosity subclass respectively. For the AGNs and hard X-ray binaries the refined classification is used (cf. Table 1)

The selection criteria were $H R 1-\delta H R 1>0.5, H R 2-$ $\delta H R 2>0.3$ with extent likelihood $L H_{\text {extent }}<50$ and count rate $>0.015 \mathrm{~s}^{-1}$. We classify these sources as class $=\mathrm{Bl}$ in Table 1 .

The sources Nos. 3, 69, 83, 84, 100, 157, 158, 160 and 242 were selected as candidate X-ray binaries. In Paper I sources with Nos. 26, 105 and 153 were rejected mainly due to the absence of time variability. For these sources radio emission was found in the radio survey of Filipović et al. (1998) and they are candidates for AGNs.

The source No. 103 (RX J0054.9 - 7226) is identified with the XTE J0055 - 724 = 1SAX J0054.9-7226 source (Marshall et al. 1998; Israel 1998). The detection of pulsations with a period of $59 \mathrm{~s}$ with BeppoSAX confirm the $\mathrm{X}$-ray binary nature of this source.

Stevens et al. (1998) identified early type emissionline stars through colour indices and $\mathrm{H} \alpha$ emission for the sources with catalogue indices $3,69,103$, and 158 .
The source No. 153 (RX J0100.7-7211) was considered to be consistent with a background AGN shining through the SMC bulge (Paper I). Sources Nos. 157 and 160 were found to coincide with detector struts and were rejected accordingly (Paper I).

\subsection{The "weaker" hard X-ray binary candidates}

Weak hard X-ray binaries are an interesting class of X-ray objects as they have been predicted to exist and their number is expected to be large especially in galaxies of low metallicity like the SMC. In previous work (Bruhweiler et al. 1987; Wang \& Wu 1992) candidates for such sources have been found and either classified as low luminosity Be systems or as background objects.

Here, we are searching for candidates of this class by applying the same selection criteria as for the strong (or higher luminosity) hard X-ray binaries as outlined in Paper I: $H R 1-\delta H R 1>0.5, H R 2-\delta H R 2>0.3$ and 
Table 4. New X-ray pulsars discovered in recent ASCA, BeppoSAX and Rossi-XTE observations of the SMC

\begin{tabular}{|c|c|c|c|c|}
\hline $\begin{array}{l}\text { Cat. } \\
\text { No. }\end{array}$ & Source name & $\begin{array}{l}\text { Pulsation } \\
\text { Period }[\mathrm{s}]\end{array}$ & $\begin{array}{l}\text { Period } \\
{[\mathrm{d}]}\end{array}$ & Ref. \\
\hline$\stackrel{98}{=}$ & $\begin{array}{l}\text { XTE J0053-724 } \\
\text { 1WGA J0053.8 - 7226 }\end{array}$ & $46.64(4)$ & $139 ?$ & $1-3$ \\
\hline \multirow[t]{2}{*}{79} & AX J0051-722 & $91.12(5)$ & $\sim 120$ & $1,4,5$ \\
\hline & XTE J0054-720 & $169.3-168.4$ & - & 1 \\
\hline$\stackrel{103}{=}$ & $\begin{array}{l}\text { XTE J0055-724 } \\
\text { 1SAX J0054.9-7226 }\end{array}$ & $59.072(1)$ & - & $5-7$ \\
\hline$\stackrel{69}{=}$ & $\begin{array}{l}\text { AX J0049-729 } \\
\text { RX J0049.1-7250 }\end{array}$ & $74.8(4)$ & - & 8,9 \\
\hline \multicolumn{5}{|c|}{$=\mathrm{RX} \mathrm{J} 0050.7-7316$} \\
\hline & AX J0058-72.0 & $280.4(3)$ & - & 14 \\
\hline \multicolumn{2}{|r|}{$\begin{aligned} & \text { 1SAX J0103.2-7209 } \\
= & \mathrm{AX} \mathrm{J0103-722}\end{aligned}$} & $345.2(1)$ & - & $\begin{array}{l}5 \\
15\end{array}$ \\
\hline & AX J0105-722 & $3.34300(3)$ & - & 21 \\
\hline & AX J0049-732 & $9.1321(4)$ & - & 22 \\
\hline & XTE J0111.2-7317 & $31.0294(7)$ & - & $23-25$ \\
\hline \multicolumn{5}{|c|}{ Previously known SMC X-ray pulsars } \\
\hline \multirow[t]{3}{*}{242} & $\mathrm{SMC} \mathrm{X}-1$ & 0.7077 & 3.892 & 16 \\
\hline & RX J0059.2-7138 & 2.76 & - & 17 \\
\hline & RX J0117.6-7330 & - & - & 18 \\
\hline$\stackrel{83}{=}$ & $\begin{array}{l}\text { RX J0051.8-7231 } \\
2 \mathrm{E} 0050.1-7247\end{array}$ & 8.9 & - & 19 \\
\hline
\end{tabular}

Refs. Corbet et al. 1998 (1), Lochner 1998b (2), Buckley et al. 1998a (3), Lochner 1998a (4), Israel et al. 1998 (5), Marshall et al. 1998 (6), Israel 1998 (7), Yokogawa \& Koyoma 1998a (8), Kahabka \& Pietsch 1998 (9), Yokogawa \& Koyoma 1998b (10), Kahabka 1998 (11), Cook 1998 (12), Schmidtke \& Cowley 1998 (13), Yokogawa \& Koyama 1998b (14), Yokogawa \& Koyoma 1998c (15), Wojdowski et al. 1998 (16), Hughes 1994 (17), Clark et al. 1997 (18), Israel et al. 1997 (19), Buckley et al. 1998b (20), Yokogawa \& Koyama 1998d (21), Imanishi et al. 1998 (22), Chakrabarty et al. (1998a) (23), Wilson \& Finger (1998) (24), Chakrabarty et al. (1998b) (25).

*) MACHO has seen a 0.708 day period. This is consistent with a rotation period of a Be star.

extent likelihood $L H_{\text {extent }}<50$. The only difference is to select objects with count rates of $<0.015 \mathrm{~s}^{-1}$, i.e. with luminosities below $\sim 310^{35} \mathrm{erg} \mathrm{s}^{-1}$ assuming a standard spectral model for the source flux (Paper I). There are 60 such objects and we tentatively classify these sources as class $=\mathrm{Bw}$. This class is a substantial fraction (25\%) of the total catalogue entries and turns out to be the class with most members.

We find 15 of these objects which coincide with Einstein detections. This may reflect that we are considerably deeper in sensitivity than the Einstein survey. Some 17 objects were found to coincide with a Simbad source. However, such a correlation could be misleading as most of the distances to the Simbad sources are too large $\left(>60^{\prime \prime}\right)$ to be considered reliable. Only three sources have a distance to a Simbad source of $<50^{\prime \prime}$ and may be considered to be identified. This is a very small fraction of all catalogued sources here.

Four sources are found close to the inner ring of the PSPC window support system and may be artifacts. A more thorough investigation of these sources appears to be required as these sources can still be real. Another 46 sources have been found inside the inner support ring of the $P S P C$ detector and are considered as firm candidates. This comprises $22 \%$ of all reliable entries in the catalogue. Sources found outside the detector ring suffer due to less accurate positions.

"Screening" of the catalog using hardness ratios derived from simulated power-law slope -0.8 spectra for hard X-ray binaries, and power-law slope -2.0 , and slope -2.6 spectra for "radio loud" and "radio quiet" AGNs respectively gives 43 firm weak hard X-ray binary candidates. Six previous hard X-ray binary candidates are consistent with AGNs and 11 candidates are consistent with either class (cf. Table 1).

Recent observations towards the SMC with $A S C A$, BeppoSAX and Rossi-XTE established 10 new X-ray pulsars in this galaxy (Table 4). Most (if not all) of them appear to be connected with a Be-type donor star. Pulsation periods in the range $3-345 \mathrm{~s}$ have been determined. This range in pulsation periods is covered by the range of pulsation periods found in the galactic Be-star X-ray binaries of $\sim 4-1500$ s (cf. van den Heuvel \& Rappaport 1987). An orbital period has been estimated only for the two systems AX J0051 - 722 and XTE J0053 - 724 with 110-120 and 139 days. The orbital period deduced for AX J0051 - 722 is in agreement with the relation between pulsation period and orbital period found by Corbet (1986), while the orbital period estimated for XTE J0053 - 724 is twice the predicted period. Five of these new X-ray pulsars may have a counterpart in our SMC X-ray catalogue. Source 98 (RX J0053.9 - 7226) has been discovered with Rossi-XTE $=$ RXTE (cf. Levine et al. 1996) in an outburst and $46.6 \mathrm{~s}$ pulsations have been found (Corbet et al. 1998). This confirms the correct classification of this source. In addition, source 79 (RX J0051.3 - 7216) may be identical with AX J0051 - 722 (Corbet et al. 1998) also confirming the correct classification. Source 89 coincides with the transient source RX J0052.9 - 7158 of Cowley et al. (1997) and AX J0051 - 73.3 (Yokogawa et al. 1998b) coincides with RX J0050.7 - 7316 (cf. Cook 1998; Cowley et al. 1997; Kahabka 1998). This source with catalog index 72 apparently both fits to the AGN and the hard X-ray binary class but it is a strong candidate for a hard X-ray binary.

The derived number of 51 hard X-ray binary candidates may be compared with the number of X-ray binaries predicted from the population synthesis calculations of Dalton \& Sarazin (1995) for the SMC. These calculations predict $46 \mathrm{X}$-ray binaries with luminosities in excess of $10^{34} \mathrm{erg} \mathrm{s}^{-1}$, the lower sensitivity limit of our SMC $\mathrm{X}$-ray survey. 
Table 5. Upper panel: Previously detected SNRs identified in the ROSAT sample, classified as SNR candidates and extended structure. The Einstein No. refers to Wang \& Wu (1992). Source radio name is given following Filipović et al. (1998)

\begin{tabular}{cccc}
\hline $\begin{array}{c}\text { Cat. } \\
\text { No. }\end{array}$ & $\begin{array}{c}\text { Einstein } \\
\text { No. }\end{array}$ & Name of SNR & $\begin{array}{c}\text { Radio } \\
\text { Source Name }\end{array}$ \\
\hline 34 & - & - & SMC B0039-7353 \\
55 & 15 & SNR 0044 - 7325 & - \\
61 & 16 & SNR 0045 - 734 & SMC B0045 - 7324 \\
68 & 22 & SNR 0047 - 735 & SMC B0047-7332 \\
77 & 24 & SNR 0049 - 736 & SMC B0049-7338 \\
140 & 44 & SNR 0057 - 724 & SMC B0057-7226 \\
189 & 52 & SNR 0103 - 726 & SMC B0103-7239 \\
191 & 53 & DEM S128 & SMC B0104-7226 \\
195 & 54 & SNR 0104 - 723 & - \\
\hline \multicolumn{4}{c}{ SNRs missed by our selection } \\
\hline 63 & 21 & SNR 0046 - 735 & SMC B0046-7333 \\
86 & 30 & SNR 0050 - 728 & - \\
90 & - & & SMC B0051 - 7254 \\
128 & 42 & SNR 0056 - 725 & - \\
148 & - & SNR 0058 - 718 & SMC B0058 - 7149 \\
177 & 50 & SNR 0101 - 724 & SMC B0101 - 7226 \\
$182 / 183$ & 51 & SNR 0102 - 723 & SMC B0102 - 7218 \\
\hline
\end{tabular}

\subsection{Supernova remnants and other extended structures}

SNRs have been identified in the SMC by work done in the radio, optical and X-ray regime (Mills et al. 1982, 1984). In the work of Ye \& Turtle (1993), some 15 SNRs and SNR candidates are detected in a $843 \mathrm{MHz}$ survey.

We applied the selection criteria likelihood of extent $L H_{\text {extent }}>50$ and $H R 1-\delta H R 1>-0.8$ in order to derive a candidate sample of SNRs and other extended structures. We find 19 objects fulfilling these criteria (including four SNRs detected by Einstein, although they have a $L H_{\text {ext }}$ below our classification threshold: sources 86, 128, 177 and 182). These sources are classified as class $=\mathrm{R}$ in Table 1 . The sources with catalogue number 95,132 , and 136 may be detector artifacts. They have the class $=\mathrm{R} / \mathrm{D}$. For 13 sources an Einstein identification has been found. We give the Einstein number and the identification for these sources in Table 5. 12 of these sources actually are known SNRs. The bright young oxygen-rich SNR 0102 - 723 (Amy \& Ball 1993) has two entries (182 and 183) in our catalog due to the merging of two pointed observations at different off-axisangles. Entry 183 is the more accurate one. An additional classified source (34) correlates with a SNR proposed by Filipović et al. (1998). We find new candidate SNRs in our X-ray survey: RX J0101.8-7249 (source 165) and RX J0112.7 - 7207 (223) were not reported before, while RX J019.4 - 7301 (245) was already detected with Einstein (BKGS 30, Bruhweiler et al. 1987), however not classified as SNR.
Table 6. Optically identified X-ray selected candidates identified with Simbad stars

\begin{tabular}{cccc}
\hline $\begin{array}{c}\text { Cat. } \\
\text { No. }\end{array}$ & $\begin{array}{c}\text { Spectral } \\
\text { Type }\end{array}$ & mag & $\begin{array}{c}\text { Distance } \\
\left({ }^{\prime \prime}\right)\end{array}$ \\
\hline 33 & F3V & 8.9 & 3 \\
44 & F6IV & 8.0 & 20 \\
109 & FV /FVI & 11.5 & 12 \\
138 & O7III & 14.1 & 52 \\
246 & A9/F0 & 7.8 & 26 \\
\hline \multicolumn{4}{c}{ Star identified in class A } \\
\hline 179 & - & 16.6 & 47 \\
\hline
\end{tabular}

We miss a few well known SNRs in the SMC with this selection. They have an extent likelihood ratio $>10$ and fulfill the criteria of being extended, suggesting we have chosen too strict an extent criterion in order to be on the secure side. It also suggests that there are still unrecognized SNRs in our sample (see discussion in Filipović et al. 1998). We list these SNRs in the lower part of Table 5.

\subsection{Foreground stars}

It is not trivial to select this sample just from the X-ray characteristics. Stars are coronal emitters with temperatures in the range of a few $10^{6}$ to $10^{7} \mathrm{~K}$. The $H R 1$ would then fall into the regime $H R 1+\delta H R 1<0.5, H R 2-$ $\delta H R 2>-0.8$ and the likelihood of extent $L H_{\text {extent }}<50$. Actually, all galactic foreground stars detected in a $8^{\circ} \times 8^{\circ}$ field centered on the SMC and observed during the RASS have values of $H R 1<0.1$ (Kahabka \& Pietsch 1993). This means that we may be too conservative in selecting stars in our sample with the criteria mentioned above.

We find 19 candidates and we classify these sources as class $=$ F in Table 1 . For seven objects, a Simbad match exists. Six of these identifications appear to be reliable as the distance to the Simbad source is $<60^{\prime \prime}$. Five of them correlate with stars of spectral type $\mathrm{O}, \mathrm{A}$ or $\mathrm{F}$ and they are given in Table 6 . Source 138 coincides with an $\mathrm{O}$ star. Assuming a conversion factor of $0.7610^{37} \mathrm{erg} \mathrm{cts}^{-1}$ we derive for the O-star in the SMC an X-ray luminosity of $10^{34} \mathrm{erg} \mathrm{s}^{-1}$ from the measured count rate. The nature of the other 14 sources remains unclear.

\subsection{Background objects and AGNS}

Background objects are selected as $H R 1-\delta H R 1>$ $0.5, H R 2+\delta H R 2<0.3$ and extent likelihood $L H_{\text {extent }}<50$. The same criterion has already been applied in Paper I in order to identify a possible sample of AGNs in the high luminosity $\mathrm{X}$-ray binary candidate sample. We find 20 sources to fulfil these criteria. Source 148 correlates with a SNR and has to 
Table 7. "Tinney" quasars found in pointings $\mathrm{X} 1$ and $\mathrm{C}$ of the SMC X-ray survey

\begin{tabular}{|c|c|c|}
\hline Cat. No. & ROSAT name & Object \\
\hline 9 & RX J0035.5 - 7201 & QJ0035 - 7201 \\
\hline 16 & RX J0036.5 - 7225 & QJ0036 - 7225 \\
\hline 17 & RX J0036.6 - 7227 & QJ0036 - 7227 \\
\hline 20 & RX J0037.3 - 7214 & QJ0037 - 7218 \\
\hline 218 & RX J0111.7 - 7250 & QJ0111 - 7246 \\
\hline 224 & RX J0112.8 - 7236 & QJ0112 - 7236 \\
\hline 238 & RX J0116.5 - 7259 & QJ0116 - 7259 \\
\hline
\end{tabular}

be removed from this class. Three sources correlate within a radius of $<60^{\prime \prime}$ with a Simbad star (either foreground or SMC). We refine and extend the classification of candidate AGNs by comparing the measured hardness ratios with the predictions from simulated power-law spectra of slope -2.0 and -2.6 . We find 53 candidates for class $=$ $\mathrm{A}$ and 62 candidates if we also consider class $=\mathrm{AB}$ in Table 1. Class = AB means a hard X-ray binary nature is also possible due to the hardness ratio criteria.

Tinney et al. (1997) present 10 quasars behind the SMC. Seven of them are covered by the fields X1 and C (cf. Table 2) and are listed in Table 7. None of these quasars was detected in the radio survey of Filipovic et al. (1998). QJ0102 - 7546, one of the three quasars not covered by our survey was detected in the RASS field (Kahabka \& Pietsch 1993).

We independently calculate the number of background sources in the analyzed field by taking the distribution of the neutral hydrogen into account. By making use of the standard $\log (N)-\log (S)$ of the soft extragalactic X-ray background (Hasinger et al. 1993) and by taking into account the absorption due to the SMC (from the H I image of Stanimirovic et al. 1999) the expected number of background sources with absorbed fluxes in excess of $10^{-13} \mathrm{erg} \mathrm{cm}^{-2} \mathrm{~s}^{-1}$ has been determined. We derive a number of 10 background sources for our covered field. As we expect to be complete for this flux limit we consider 10 background sources as the lower limit. The lower end of the flux distribution extends to $10^{-14} \mathrm{erg} \mathrm{cm}^{-2} \mathrm{~s}^{-1}$. In case of completeness we expect to detect 519 background sources. The fact that we classify $53-62$ sources as background sources is consistent with these numbers.

\section{Conclusions}

We performed an X-ray survey of a $8.95 \mathrm{deg}^{2}$ field in the direction of the Small Magellanic Cloud. We detect 248 point-like and moderately extended sources. Using criteria established here, six sources were classified as supersoft sources, 51 as hard X-ray binary candidates, 19 as supernova remnants, 19 as candidate foreground stars and 53 as candidate background AGNs. These are
$60 \%$ of all catalog entries. The number of hard X-ray binaries agrees with the numbers predicted from population synthesis calculations for luminosities in excess of $10^{34} \mathrm{erg} \mathrm{s}^{-1}$. Assuming the standard $\log (N)-\log (S)$ of the soft extragalactic X-ray background we estimate that in our field are 10 background AGNs with fluxes in excess of $10^{-13} \mathrm{erg} \mathrm{cm}^{-2} \mathrm{~s}^{-1}$ and 519 background AGNs with fluxes in excess of $10^{-14} \mathrm{erg} \mathrm{cm}^{-2} \mathrm{~s}^{-1}$. We propose three new SNR candidates.

Acknowledgements. This research was supported in part by the Netherlands Organization for Scientific Research (NWO) through Spinoza Grant 08-0 to E.P.J. van den Heuvel. P.K. thanks E.P.J. van den Heuvel for stimulating discussions and $\mathrm{X}$. Li for reading the manuscript. S. Stanimirovic is thanked for providing the $\mathrm{HI}$ image of the SMC. Part of the work has been performed during the stay of P.K. at the Max-Planck-Institut für extraterrestrische Physik in Garching. The ROSAT project is supported by the MaxPlanck-Gesellschaft and the Bundesministerium für Forschung und Technologie (BMFT). This research made use of the Simbad data base operated at CDS, Strasbourg, France. We thank the referee for useful comments and suggestions to improve this work.

\section{References}

Amy S.W., Ball L., 1993, ApJ 411, 761

Bruhweiler F.C., Klinglesmith III., Gull T.R., et al., 1987, ApJ 317,152

Buckley D.A.H., Coe J., Stevens B., et al., 1998a, IAU Circ. No. 6789

Buckley D.A.H., Stevens B., Coe J., et al., 1998b, MNRAS (submitted)

Caplan J., Ye T., Deharveng L., et al., 1996, A\&A 307, 403

Cash W., 1979, ApJ 228, 939

Chakrabarty D., Levina A.M., Clark G.W., Takeshima T., 1998a, IAU Circ. No. 7048

Chakrabarty D., Ozaki M., Paul B., Yokogawa J., 1998b, IAU Circ. No. 7062

Clark G.W., Remillard R.A., Woo J.W., 1997, ApJ 474, L111

Coarer E., Rosado M., Georgelin Y., et al., 1993, A\&A 280, 365

Cook K., 1998, IAU Circ. No. 6860

Corbet R.H.D., 1986, in: The Evolution of Galactic X-ray Binaries, Trümper J. et al. (eds.). Reidel Pub. Co., p. 63

Corbet R., Marshall F.E., Lochner J.C., et al., 1998, IAU Circ. No. 6803

Cowley A.P., Schmidtke P.C., McGrath T.K., et al., 1997, PASP 109, 21

Cruddace R.G., Hasinger G., Schmitt J.H.M.M., 1988, in: Astronomy from Large Databases, Murtagh F., Heck A. (eds.), p. 177

Dalton W.W., Sarazin C.L., 1995, in: High Mass X-ray Binary Modeling: The Magellanic Cloud Populations (preprint)

Davies R.D., Elliot K.H., Meaburn J., 1976, Mem. R. Astr. Soc. 81,89

Filipović M.D., Pietsch W., Haynes R.F., et al., 1998, A\&AS 127,119 
Filipović M.D., White G.L., Haynes R.F., et al., 1997, A\&AS 121,321

Hasinger G., Burg R., Giacconi R., et al., 1993, A\&A 275, 1

Hughes J.P., 1994, ApJ 427, L25

Imanishi K., Yokogawa J., Koyama K., 1998, IAU Circ. 7040

Inoue H., Koyama K., Tanaka Y., 1983, in: IAU Symposium 101, Supernova Remnants and Their X-ray Emission, Danziger J., Gorenstein P. (eds.). Dordrecht: Reidel, p. 535 Irwin J.A., Sarazin C., 1998, ApJ 499, 650

Israel G.L., 1998, IAU Circ. 6822

Israel G.L., Stella L., Angelini L., et al., 1997, ApJ 484, L141

Israel G.L., Stella L., Campana S., et al., 1998, IAU Circ. 6999

Kahabka P., Pietsch W., 1993, in: Lecture Notes in Physics 416, New Aspects of Magellanic Cloud Research, Baschek B., Klare G., Lequeux J. (eds.), p. 71

Kahabka P., 1998, IAU Circ. No. 6854

Kahabka P., Pietsch W., Hasinger G., 1994, A\&A 288, 538

Kahabka P., Pietsch W., 1996, A\&A 312, 919 (Paper I)

Kahabka P., Pietsch W., 1998, IAU Circ. No. 6840

Levine A.M., Bradt H., Cui W., et al., 1996, ApJ 469, L33

Lochner J.C., 1998a, IAU Circ. No. 6858

Lochner J.C., 1998b, IAU Circ. No. 7007

Lochner J.C., Marshall F.E., Whitlock L.A., et al., 1998, IAU Circ. No. 6814

Marshall F.E., Lochner J.C., Santangelo A., et al., 1998, IAU Circ. 6818

Meyssonnier N., Azzopardi M., 1993, A\&AS 102, 251

Mills B.Y., Little A.G., Durdin J.M., et al., 1982, MNRAS 200, 1007

Mills B.Y., Turtle A.J., Little A.G., et al., 1984, Aust. J. Phys. 37,321

Pfeffermann E., Briel U.G., Hippmann H., et al., 1986, Proc.
SPIE 733, 519

Rubio M., Lequeux J., Boulanger F., et al., 1993a, A\&A 271, 1

Rubio M., Lequeux J., Boulanger F., et al., 1993b, A\&A 271, 9

Seward F.D., Mitchell M., 1981, ApJ 243, 736

Schmidtke P.C., Cowley A.P., 1998, IAU Circ. No. 6880

Stanimirovic S., Staveley-Smith L., Dickey J.M., et al., 1999, MNRAS 302, 417

Staveley-Smith L., Sault R.J., Hatzidimitriou D., et al., 1997, MNRAS 289, 225

Stevens J.B., Coe M.J., Buckley D.A.H., 1998, MNRAS (submitted)

Tinney C.G., Da Costa G.S., Zinnecker H., 1997, MNRAS 285, 111

Trümper J., 1983, Adv. Space Res. 2, No. 4, 241

van den Heuvel E.P.J., Rappaport S.A., 1987, in: Physics of Be Stars, Slettebak A., Snow T.P. (eds.). Cambridge Univ. Press, p. 290

Wang Q., Wu X., 1992, ApJS 78, 391

Wilson C.A., Finger M.H., 1998, IAU Circ. No. 7048

Wojdowski P., Clark G.W., Levine A.M., et al., 1998, ApJ (in press)

Ye T., Turtle A.J., 1993, in: Lecture Notes in Physics 416, New Aspects of Magellanic Cloud Research, Baschek B., Klare G., Lequeux J. (eds.), p. 167

Yokogawa J., Koyama K., 1998a, IAU Circ. No. 6835

Yokogawa J., Koyama K., 1998b, IAU Circ. No. 6853

Yokogawa J., Koyama K., 1998c, IAU Circ. No. 7009

Yokogawa J., Koyama K., 1998d, IAU Circ. No. 7028

Zimmermann H.U., Becker W., Belloni T., et al., 1994, MPE report 257 
Table 1. ROSAT PSPC Point Source Catalogue of the SMC

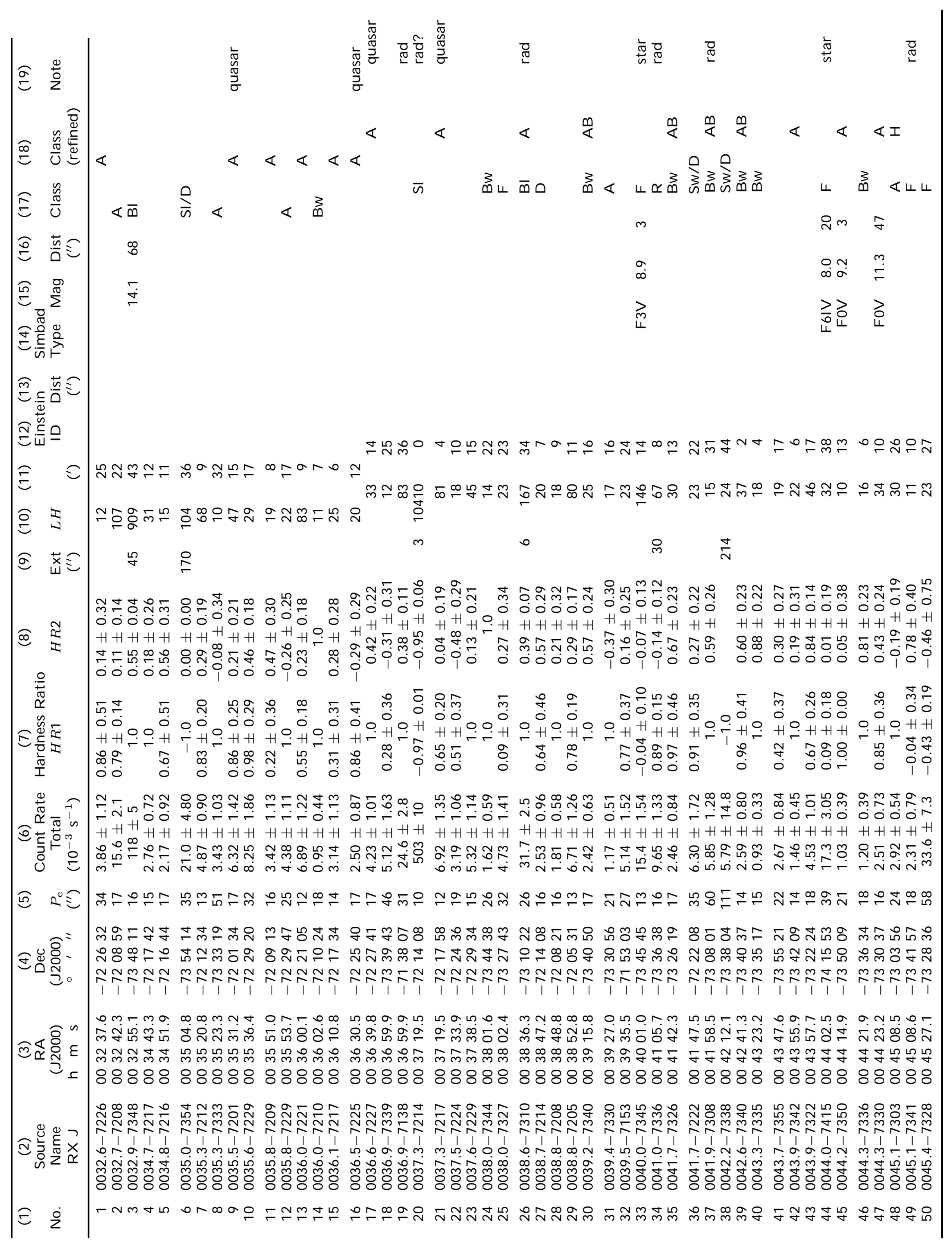


Table 1. continued

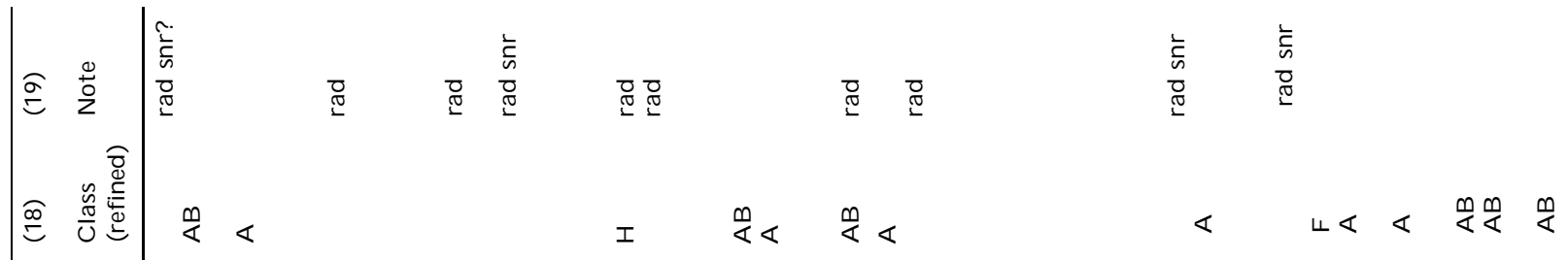

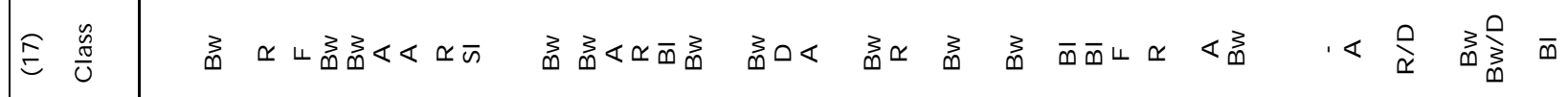

$$
\begin{aligned}
& \text { 矛 }
\end{aligned}
$$

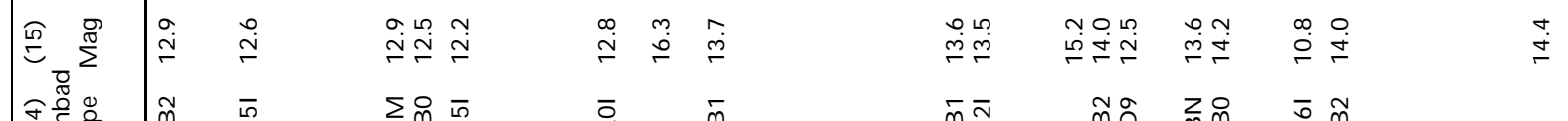

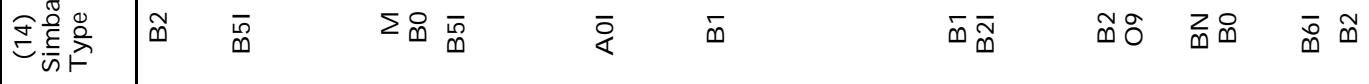

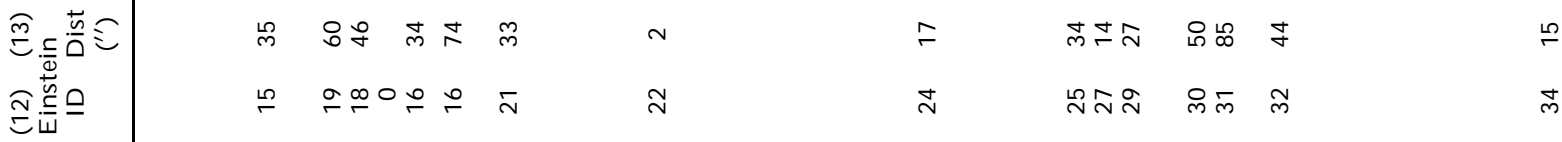

$$
\begin{aligned}
& \text { 凷 }
\end{aligned}
$$

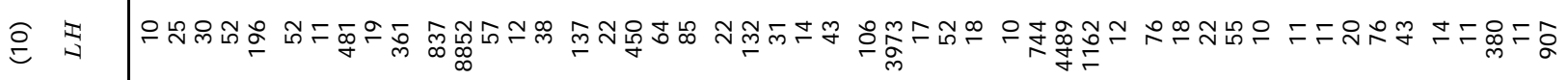

$$
\begin{aligned}
& \text { a }
\end{aligned}
$$

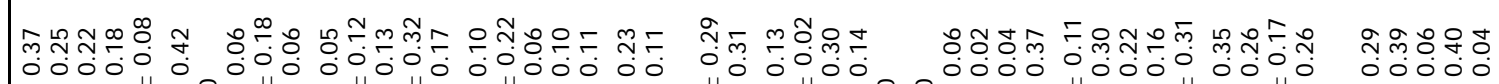

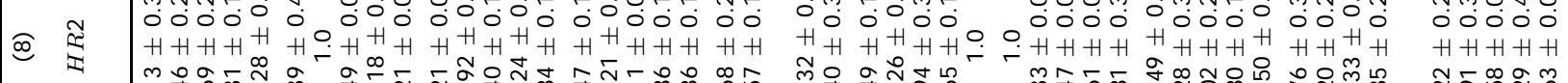

$$
\begin{aligned}
& \text { mo엄ำ }
\end{aligned}
$$

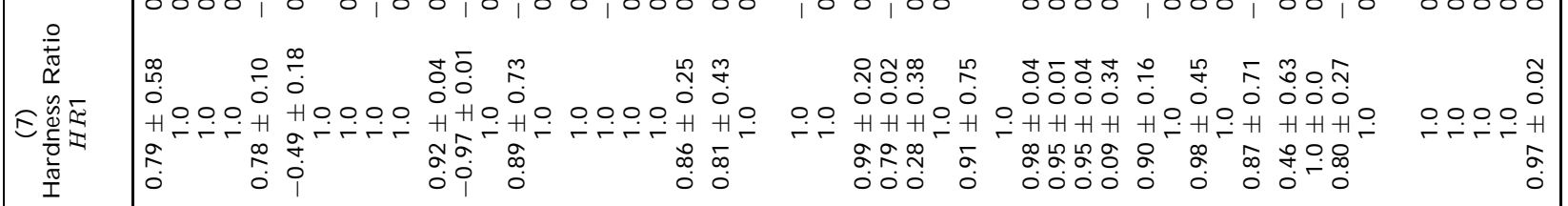

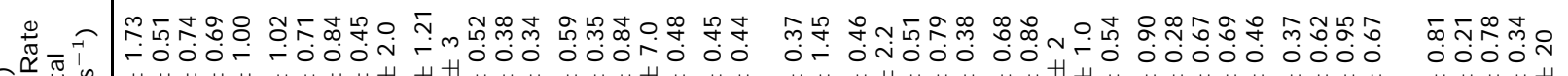

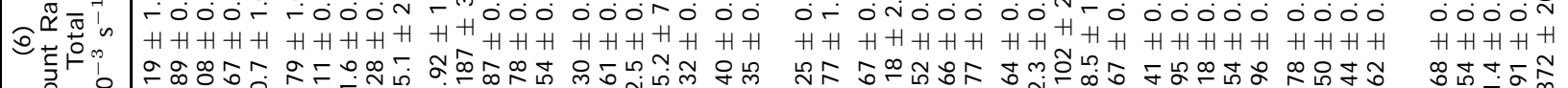

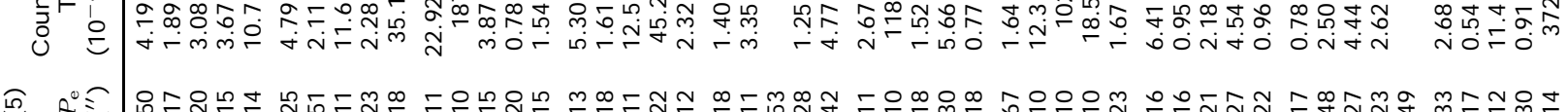

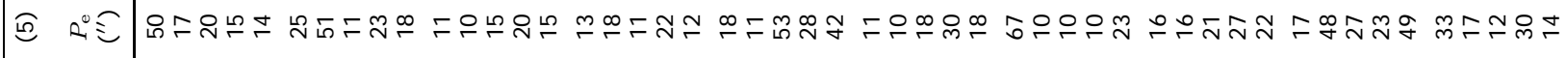

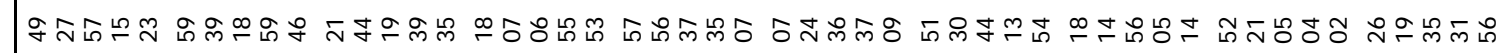

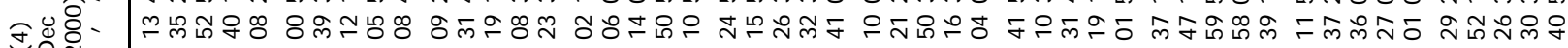


Table 1. continued

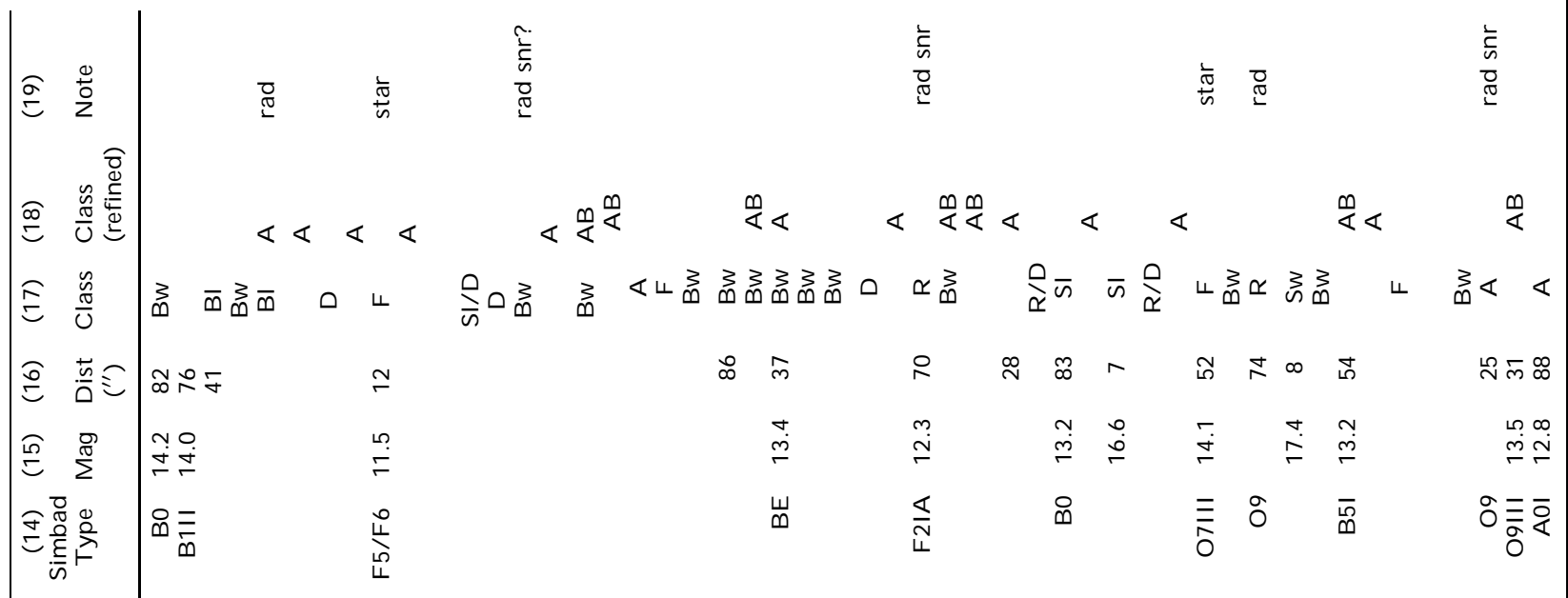

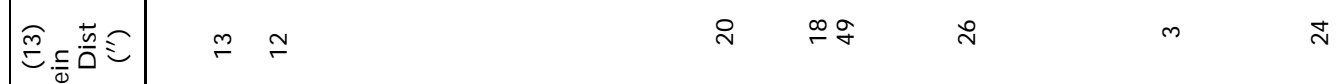

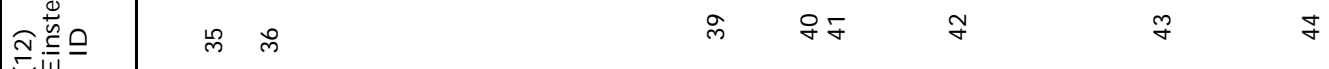

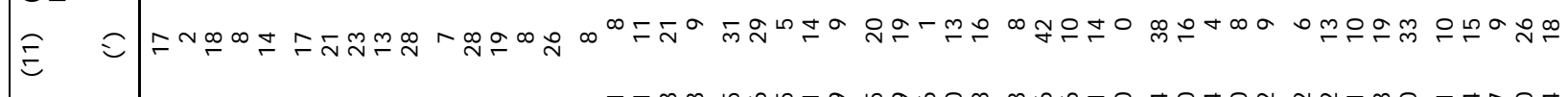

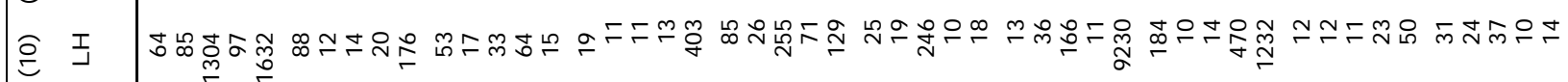

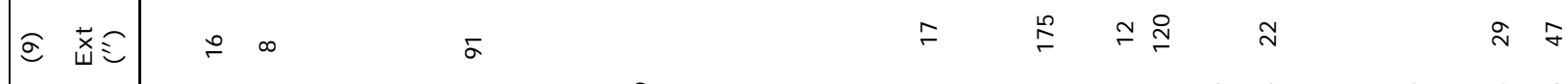

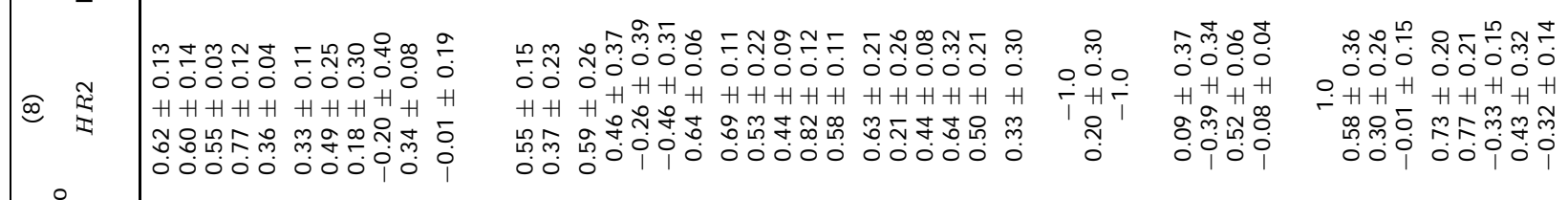

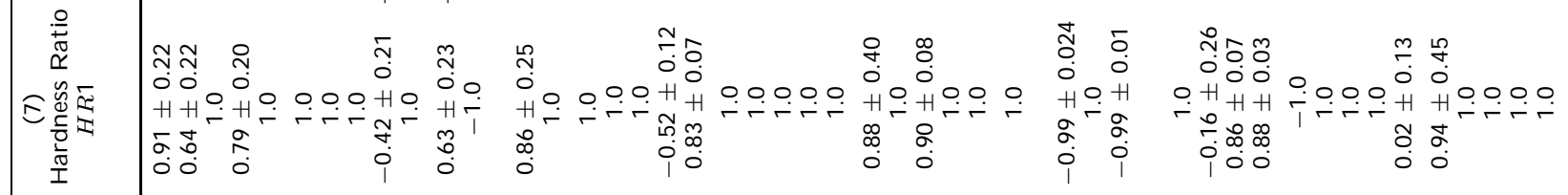

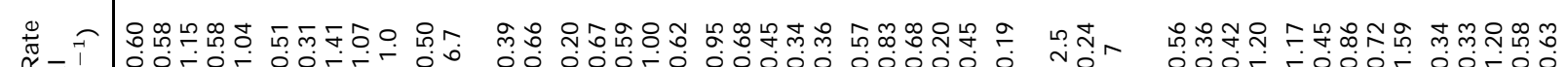

$$
\begin{aligned}
& \text { Q } \\
& \text { U }
\end{aligned}
$$

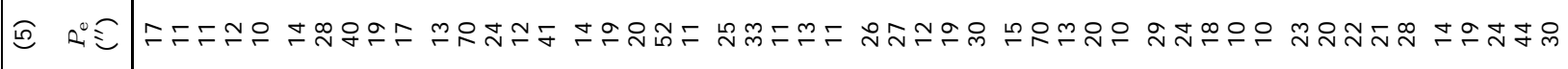

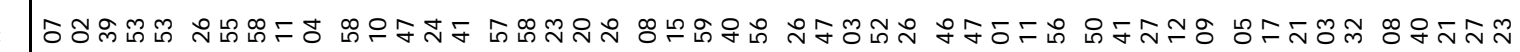

$$
\begin{aligned}
& \text { 子 } \\
& \text { g. } 35 N \text {. }
\end{aligned}
$$

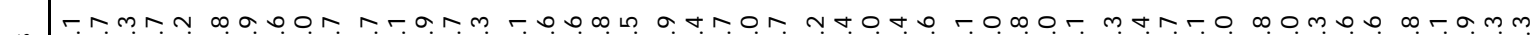

$$
\begin{aligned}
& \text { «㲅 }
\end{aligned}
$$

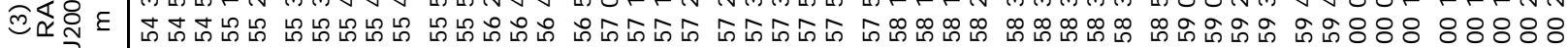

$$
\begin{aligned}
& =\text { _ } 8888888888888888888888888888888888888888880^{-1} \sigma^{-1} \sigma^{-1} \sigma^{-1}
\end{aligned}
$$

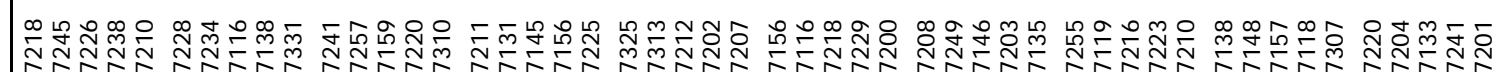

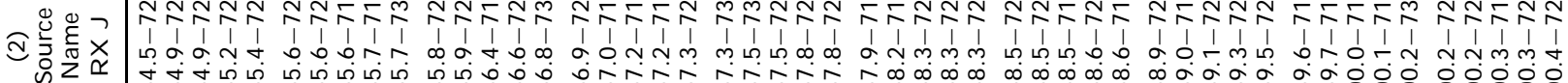

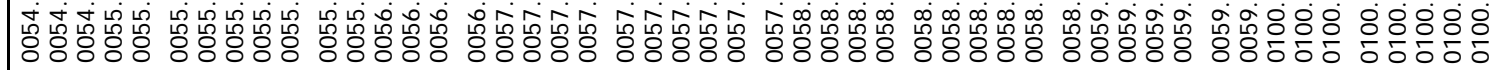

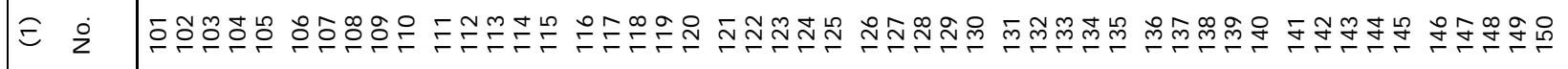


Table 1. continued

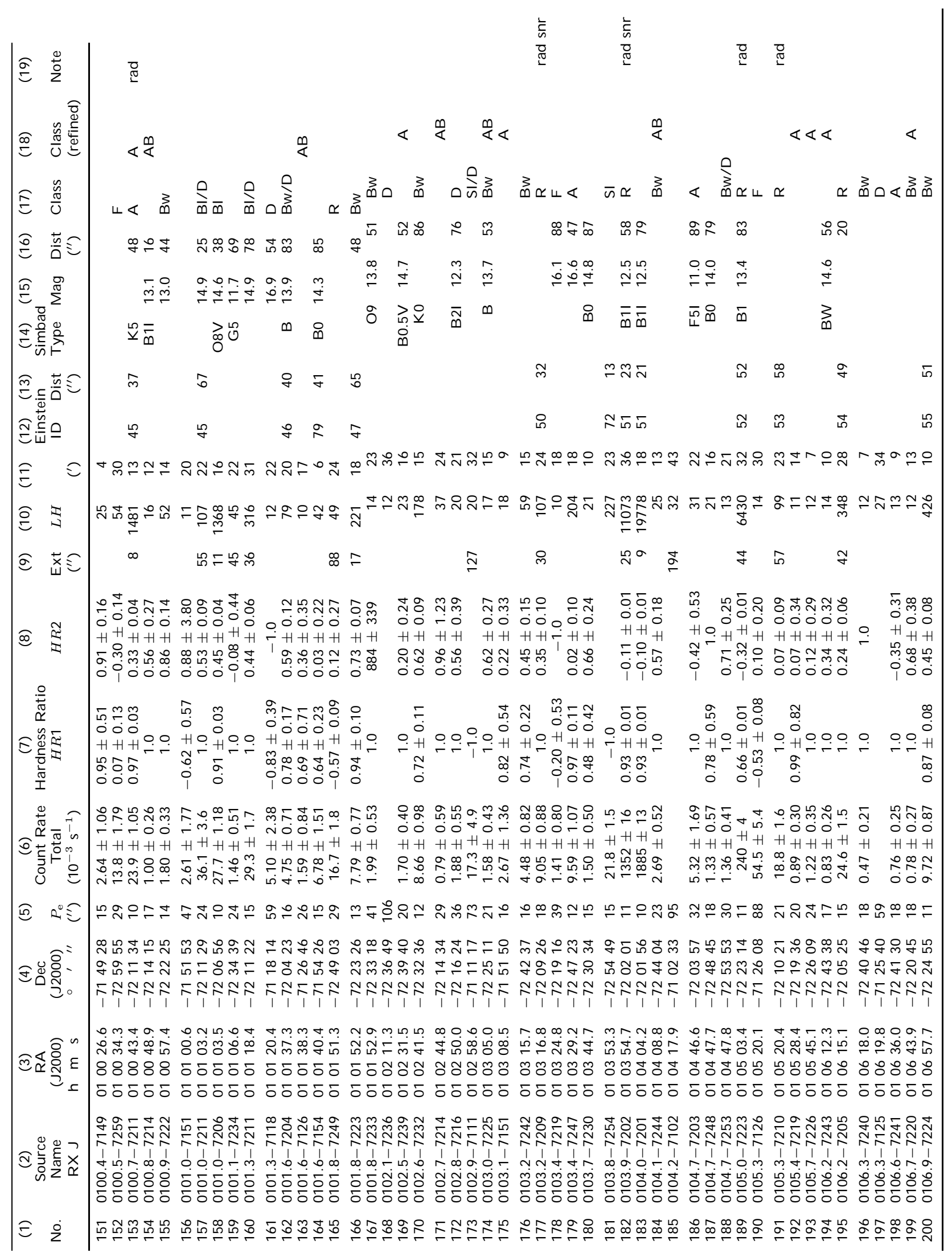


Table 1. continued

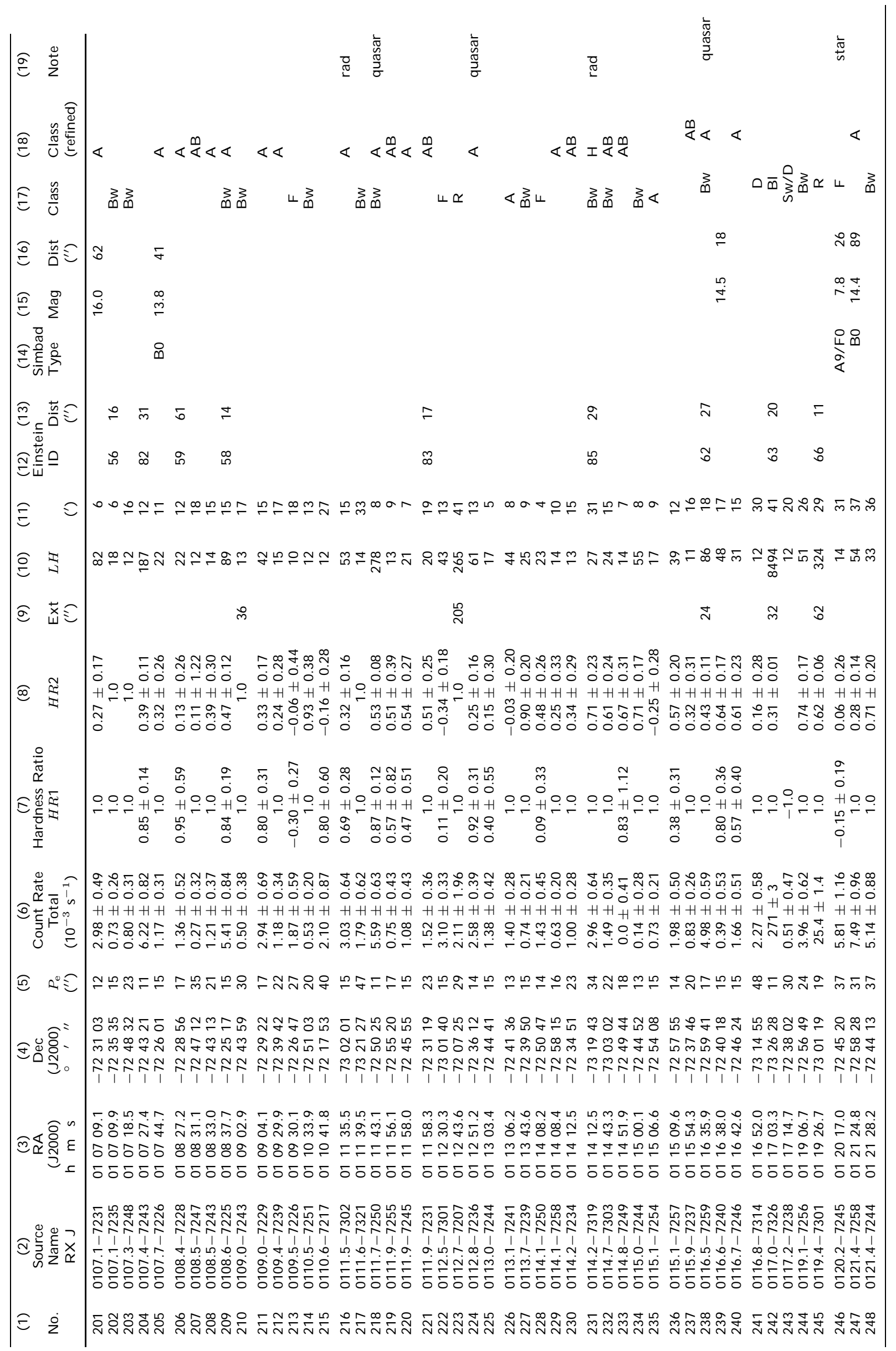

